\title{
ECG Identification Based on Wavelet Transform
}

\author{
WANG Wei-Quan ${ }^{1}$, LU Pan ${ }^{2, *}$, LIN Jia-Lun ${ }^{1}$, ZHANG Jin ${ }^{1}$ \\ 1.Department of Network management center, Hainan Medical University, Hainan, 571199, China \\ 2. School of Computer Science and Engineering, South China University of Technology, \\ Guangzhou 510006, China \\ *email: 1907961382@qq.com
}

\section{Keywords: Electrocardiography Biometric Identification Wavelet Transform MATLAB}

\begin{abstract}
ECG signal, a kind of internal characteristics of the human body, which reflects the electrical activity of the heart, has multiple excellent features such as difficult to duplicate, hard to forge, unique, stable and so on. In this paper, the identification of ECG signal is studied based on the theory of wavelet transform. First of all, we introduce the present situation of biometric identification, and then carry out a detailed deduction of wavelet transform to be used and finally simulate the test in MATLAB. The analysis of experimental results shows that the accuracy of identification is high and satisfactory and it is feasible and practical.
\end{abstract}

\section{Introduction}

With the continuous development of society and Information Technology, Identification is playing a more and more important part in our everyday life. Meanwhile, the requirements of efficiency and accuracy of identification in each field are increasing, so that the certification effects of the conventional authentication approaches which expose more and more their drawbacks in practice are not satisfactory. The traditional identification approaches including password, credentials and so on which have defects such as easy to copy, forget, lose cannot satisfy the demand of information safety. At this time, the emergence of a new method of authentication named Biometric Identification appears to effectively make up the deficiency of traditional approaches and it has been widely used in the criminal investigation, financial transactions, e-commerce, airport security and homeland security etc. As its name suggests, Biometric Identification Technology achieves the aim of authentication by using specific biological features and now the common biological features include fingerprint, iris, voice, gait, palm print, etc.

Numerous studies have shown that electrocardiograph is unique, easy to collect and not easy to copy, providing a new identification approach and become a research hot spot. The ECG signal which reflects the electric potential signal of heartbeats is collected from human body surface. Different people have different ECG definitely; even the same person may have different ECG signals in various conditions such as heart disease. This kind of dynamic characteristic of ECG is different from the static features such as fingerprint, palm print etc. So it's not easy to be imitated or used by others which further improves the security of identification, but also increases the complexity of recognition. 


\section{Proposed Method}

ECG signal is from the weak electrical signal of cardiomyocyte and because of weakness itself and signal-acquisition equipment interfered by outside situation, the collected signal is interfered by different levels of noise which mainly includes electromagnetic noise, baseline drift, EMG interference etc. Therefore, the noise reduction process of ECG signal is the key to ensure the accuracy of identification. And the commonly used de-noising methods include Fourier transform and Wavelet transform. For the irregular signal like ECG, the Fourier transform which based on global optimization technique, however, cannot exert its greatest advantage. However, As for Wavelet transform, we can obtain high frequency signal with short time interval and obtain low frequency signal with long time interval, so it's an ideal tool for the analysis of ECG. To ensure the accuracy of ECG identification, based on the theory of Wavelet transform we preprocess and analyze the ECG signal and implement authentication in this paper.

The continuous wavelet transform is defined as: Assume $f(x)$ is a square integral function, that is $\mathrm{f}(x) \ni \mathrm{L}^{2}(R), \mathrm{L}^{2}(R)$ represents real space with square integral. When and only when the Fourier transform $f(\omega)$ satisfies the condition (1), at this point we call $\mathrm{f}(\mathrm{x})$ as a basic wavelet transform function or mother wavelet function.

$$
\int_{R} \frac{|f(\omega)|^{2}}{\omega} d \omega<\infty
$$

After translation and dilation transformation to the basic wavelet transform function $\mathrm{f}(\mathrm{x})$, we can obtain the following wavelet series.

$$
f_{a, b}(x)=\frac{1}{\sqrt{|a|}} f\left(\frac{x-b}{a}\right) a, b \in R, a \neq 0
$$

Wherein: $\mathrm{a}$ and $\mathrm{b}$ are factors of flexibility and parallelism.

Set $g(t)$ for any square integral function, then its continuous wavelet transform is:

$$
W T_{g}(a, b)=\frac{1}{\sqrt{|a|}} \int_{-\infty}^{+\infty} g(t) f^{*} \frac{t-b}{a} d t=<g t\left(\mathfrak{f}_{a b} t \quad b\right.
$$

In the formula (3), symbol a is a scale factor and the value is greater than 0 ; $b$ is the factor of parallelism and the value can be positive or negative. $\langle\mathrm{x}(\mathrm{t}), \mathrm{y}(\mathrm{t})\rangle$ represents inner product, that is:

$$
<x(t), y(t)>=\int x(t) y^{*}(t) d t
$$

Wherein: the asterisk indicates conjugate.

The continuous wavelet inverse transformation of formula (3) is:

$$
g(t)=\frac{1}{C_{f}} \int_{R^{+}} \int_{R} \frac{1}{a^{2}} W T_{g}(a, b) f\left(\frac{t-b}{a}\right) d a d b
$$

Considering that the redundant parameters in the continuous transform can affect the performance of computation, we discretize the time parameter and the function to be processed using the following rules: 


$$
\begin{aligned}
& a=a_{0}^{m}, a_{0}>0, m \in Z \\
& b=n b_{0} a_{0}^{m}, b \in R, n \in Z
\end{aligned}
$$

After discretization by formula (6), the primitive function $\psi_{a, b}(t)$ is converted into:

$$
\psi_{m, n}(t) a_{0}^{-\frac{m}{2}}\left(a_{0}^{-m} t-n b_{0}\right)
$$

Therefore, the discrete wavelet transform (DWT) of function $\mathrm{g}(\mathrm{t})$ is:

$$
W T_{g}(m, n)=\int_{R} g(t) \psi_{m, n}^{*}(t) d t
$$

Where in: $\psi_{m, n}(t)=2^{-\frac{m}{2}} \psi\left(2^{-m} t-n\right)$

In the preprocessing of ECG signal, assume $s(t)$ is the original signal collected by ECG signal-acquisition equipment, and then the signal with noise can be described as follows:

$$
s(t)=g(t)+\sigma * \text { noise }(t)
$$

In the formula above, $\sigma$ indicates the standard deviation of noise factor and $g(t)$ is the useful signal. And from the name of function noise $(t)$ we can see that it indicates the noise information obviously.

Based on the methods from formula (1) to (8), we can preprocess the collected original ECG signal and achieve identification after that. The main purpose of preprocessing is that eliminate the noise with high or low frequency to make sure the accuracy of identification can be improved. In preprocess of de-noising, we firstly decompose the signal s (t) using wavelet (Figure 1), and then threshold-value filtering, finally, reconstruct signal. After preprocessing, we can obtain signal h $(\mathrm{t})$ with no or very little noise.

Wavelet signal decomposition process is shown below:

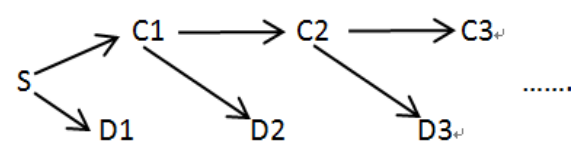

Figure 1 Schematic diagram of signal wavelet decomposition

\section{Experimental Results}

Based on the methods proposed in the Proposed Method section, we simulate the ECG signal preprocessing and the identification results in MATLAB.

\subsection{ECG Preprocessing}

By simulating in MATLAB, we obtain the ECG signal waveforms before and after processing. 


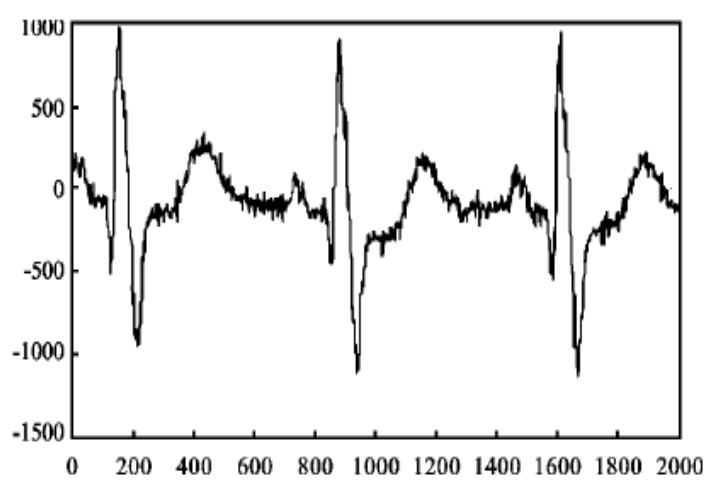

Figure 2 ECG with noise

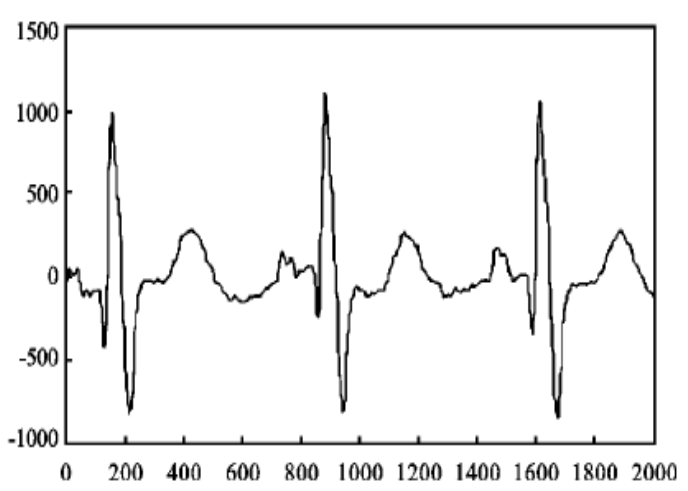

Figure 3 ECG after preprocessing

Figure 2 shows the original ECG signal before preprocessing and it can be seen intuitively that the signal waveform is very rough and not smooth which contains a lot of noise. After processing the signal using methods proposed above, we can obtain waveform in Figure 3 through which we can analyze that the ECG signal becomes much smoother and is close to the ideal state of ECG waveform at this time. What's more, according to comparative analysis of Figure 2 and Figure 3 can be drawn that the signal waveform does not have the obvious distortion phenomenon using the preprocessing method proposed above which contributes to improve the accuracy of identification in the follow-up experiment.

\subsection{ECG identification results and analysis}

In the process of identifying the ECG signals with normal heart rate, the accuracy of identification varies with different number of selected heartbeat periods at each time. After repeatedly simulation test in MATLAB, test results are shown in Figure 4 in which the horizontal axis of $n$ indicates the heartbeat cycle number and the vertical axis represents the corresponding identification accuracy.

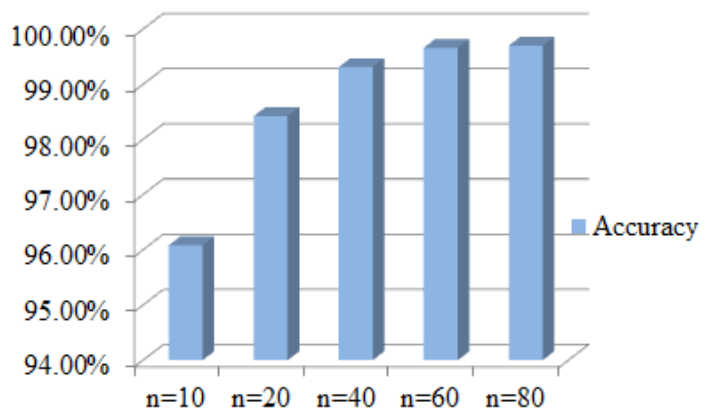

Figure 4 Accuracy varies with heartbeat cycle diagram

From Figure 4, when the number of heartbeat cycle increases in the processing of recognition and the accuracy also corresponding increase. When $\mathrm{n}$ equals 80 , the corresponding accuracy reaches $99.70 \%$ and when $n$ equals 10 , the accuracy can also reach $96.80 \%$. What's more, from the Figure we can know that the accuracy will gradually tend to be stable when the n value is more and more large.

At the same time, several simulation tests are carried out for ECG signals with different degrees of arrhythmia. And the corresponding results are shown in the table below named Table 1. Also, the corresponding three-dimensional column diagram is shown in Figure 5.

\begin{tabular}{cccccc}
\hline $\begin{array}{c}\text { Type of } \\
\text { ECG }\end{array}$ & $\begin{array}{c}\text { Test } \\
\text { Size }\end{array}$ & $\begin{array}{c}\text { Upper } \\
\text { Accuracy }\end{array}$ & $\begin{array}{c}\text { Lower } \\
\text { Accuracy }\end{array}$ & $\begin{array}{c}\text { Final } \\
\text { Accuracy }\end{array}$ & $\begin{array}{c}\text { Percentage } \\
\text { of Rejections }\end{array}$ \\
\hline Short-term Normal ECG (STNE) & 36360 & $76.29 \%$ & $77.48 \%$ & $88.32 \%$ & $27.89 \%$ \\
Short-term ECG with Arrhythmias (STEA) & 91749 & $80.01 \%$ & $79.21 \%$ & $90.64 \%$ & $25.42 \%$ \\
Long-term Normal ECG (LTNE) & 62565 & $69.42 \%$ & $69.02 \%$ & $86.55 \%$ & $39.56 \%$ \\
Long-term ECG with Arrhythmias (LTEA) & 232749 & $61.58 \%$ & $62.37 \%$ & $87.08 \%$ & $43.29 \%$ \\
\hline
\end{tabular}


Table 1 the result table of Arrhythmia signals

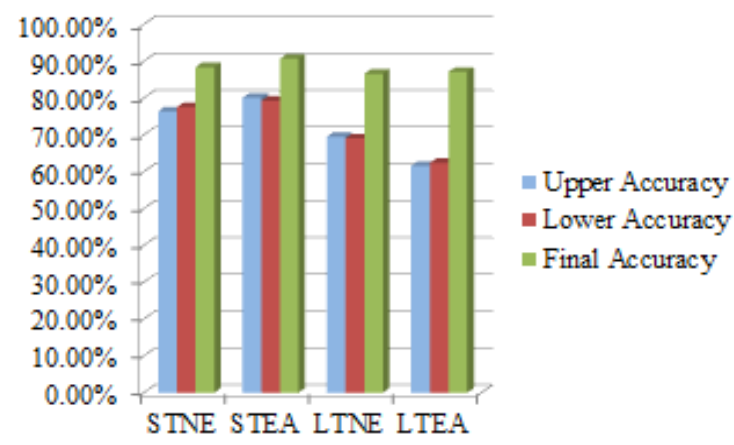

Figure 5 Test accuracy rate change diagram

According to the comparative analysis of accuracy under different conditions can be seen that we can achieve satisfactory recognition accuracy about $86 \%$ to $90 \%$ no matter it is the short-term arrhythmia or long-term arrhythmia ECG signal. Therefore, for the different types of ECG whether normal or with arrhythmia, the identification accuracy is satisfactory by using the method proposed in the paper.

\section{Conclusion}

ECG signal is a new biometric identification technology which is unique and difficult to be disguised. With the popularity and miniaturization of ECG signal acquisition devices, the Identification based on ECG will be adopted in more and more applications. Based on the theory of wavelet transform, the methods of preprocessing and identify recognition have been investigated in this paper. The experimental data shows that this method can achieve high and satisfactory accuracy, so it has a certain reliability and practicability. However, as for the problems exist in ECG identification such as the same person may have different ECG signal because of physical condition or mental state, further researches are still needed.

\section{Acknowledgement}

This work is supported by Hainan Natural Fund Project(20156229), and Hainan Medical University Scientific Research and Cultivation Fund Project(HY2014-004).

\section{References}

[1]Tian Jie, Yang xin. Theory and application of biometric identification technology[M], Beijing. Publishing House of Electronics Industry, 2005

[2]Yang Lei.Study on Human Identification Algorithm Based on ECG[D].Hangzhou: Hangzhou Dianzi University,2011

[3] W Boles, "A Security System Based on Human Iris Identification Using Wavelet Transform",in Proc. First Int. Conf Knoi/ledge-Based Intelligent Electron. Syst.,New York,1997

[4]Chiu C C , Chuang C M, Hsu C Y. A novel personal identity verification approach using a discrete wavelet transform of the ECG signal[C].Proceeding of 2008 International Conference on Multimedia and Ubiquitous Engineering Washington D C : IEEE,2008: 201-206

[5]Yuhong Liu, Xingliang Xiong, Zhifang Wang, Ying Wang, Hong Li, Jianbo Li, Zhengxiang Xie. Theories and techniques for bi -valuating image with parameters and their applications [J]. Application Research of Computers. 2008, 25(1):277-280. 